\title{
On maximal inequalities via comparison principle
}

Cloud Makasu*

\section{"Correspondence:}

cmakasu@uwc.ac.za

Department of Mathematics and Applied Mathematics, University of the Western Cape, Private Bag X17, Bellville 7535, Cape Town,

South Africa

\begin{abstract}
Under certain conditions, we prove a new class of one-sided, weighted, maximal inequalities for a standard Brownian motion. Our method of proof is mainly based on a comparison principle for solutions of a system of nonlinear first-order differential equations.
\end{abstract}

Keywords: Brownian motion; comparison principle; nonlinear differential equations

\section{Introduction}

Let $B=\left(B_{t}\right)_{t \geq 0}$ be a standard Brownian motion starting at zero, and let $\tau$ be any stopping time for $B$ with finite expectation. It is well known that

$$
\mathbf{E}\left[\max _{0 \leq t \leq \tau}\left|B_{t}\right|\right] \leq \sqrt{2 \mathbf{E}[\tau]}
$$

where $\sqrt{2}$ is the best possible constant.

The one-sided maximal inequality (1) is proved in Dubins et al. [1]; see also [2]. Recently, using a similar method of proof to Dubins et al. [2], the present author has proved the following weighted maximal inequality for a standard Brownian motion.

Theorem 1 Let $B=\left(B_{t}\right)_{t \geq 0}$ be a standard Brownian motion starting at $x \geq 0$. Then the weighted maximal inequality

$$
\mathbf{E}_{x}\left[\left|B_{\tau}\right|\left(\max _{0 \leq t \leq \tau}\left|B_{t}\right|\right)\right] \leq 2 \mathbf{E}_{x}[\tau]+\frac{3}{2} x^{2}
$$

holds for any stopping time $\tau$ for $B$ satisfying $\mathbf{E}_{x}[\tau]<\infty$. The constants 2 and $3 / 2$ in (2) are the best possible.

The proof of the above theorem is essentially based on the next lemma, which can easily be proved using similar arguments to [2].

Lemma 2 Assume that $H(\cdot)$ is a positive, continuous and differentiable function defined on $[a, \infty)$ with $a \geq 0$ such that $H(a) \geq 0$. Consider the following optimal stopping problem:

$$
u(x, y):=\sup _{\tau} \mathbf{E}_{x, y}\left[X_{\tau} H\left(Y_{\tau}\right)-c \tau\right] \quad(c>0),
$$

(c) 2015 Makasu. This article is distributed under the terms of the Creative Commons Attribution 4.0 International License (http://creativecommons.org/licenses/by/4.0/), which permits unrestricted use, distribution, and reproduction in any medium, provided you give appropriate credit to the original author(s) and the source, provide a link to the Creative Commons license, and indicate if changes were made. 
where the supremum is taken over all stopping times $\tau$ for B satisfying $\mathbf{E}_{x, y}[\tau]<\infty, X_{t}=\left|B_{t}\right|$, and $Y_{t}=\left(\max _{0 \leq s \leq t} X_{s}\right) \vee y$ with $0 \leq x \leq y$ given and fixed.

Then:

(a) the value function $u(x, y)$ is of the form

$$
u(x, y)= \begin{cases}c x^{2}+x H(y)-2 c x g_{*}(y)+c\left(g_{*}(y)\right)^{2}, & g_{*}(y)<x \leq y \\ x H(y), & 0 \leq x \leq g_{*}(y)\end{cases}
$$

(b) the optimal stopping time $\tau_{*}$ is of the Azéma-Yor type given by

$$
\tau_{*}=\inf \left\{t>0: X_{t} \leq g_{*}\left(Y_{t}\right)\right\}
$$

where $X_{t}=\left|B_{t}\right|$ and $y \mapsto g_{*}(y)$ is the maximal solution of the first-order nonlinear differential equation

$$
g^{\prime}(y)=\frac{1}{2 c}\left(\frac{y H^{\prime}(y)}{y-g(y)}\right)
$$

under the condition $0<g(y)<y$.

Remark 3 A characterization of optimal stopping boundaries $y \mapsto g_{*}(y)$ in terms of the maximal solution of a nonlinear first-order differential equation for a general class of diffusion processes is given in Peskir [3].

Remark 4 For the existence of the maximal solution $y \mapsto g_{*}(y)$ of (4), note that the righthand side of (4) is nondecreasing in $g(\cdot)$ provided that $H^{\prime}(\cdot)>0$.

It must be noted that the maximal solution of (4) can only be given explicitly in a few special instances. In the case when $H(y)=y$ defined on $[0, \infty)$, then

$$
g_{*}(y)=\frac{1}{2}\left(1+\sqrt{1-\frac{2}{c}}\right) y
$$

for $0<y<\infty$ and $c>2$. This leads to the proof of the weighted maximal inequality (2). In the general case, the maximal solution $y \mapsto g_{*}(y)$ of (4) cannot be given explicitly. This general case is dealt with in this paper. As a result, we obtain a generalization of the one-sided, weighted, maximal inequality (2) and an extension of the well-known maximal inequality (1).

Throughout the paper, we shall assume the following:

(A) $H$ is a positive, continuous, and differentiable function defined on $[a, \infty)$ with $a \geq 0$ such that $H(a) \geq 0$ and there exist constants $K>0$ and $M \geq 0$ such that

$$
\left|y H^{\prime}(y)\right| \leq K y+M
$$

holds for all (some) $y>a$. 
The condition (A) plays a vital role in this paper. It is useful in establishing a two-sided, explicit estimate of the maximal solution $y \mapsto g_{*}(y)$ of (4). This estimate is obtained via a comparison principle (see for instance [4-7] etc.), which is an important tool in the theory of ordinary differential equations.

Assume that condition (A) holds. Consider the nonlinear equation (4), and an auxiliary comparison nonlinear equation of the form

$$
h^{\prime}(y)=\frac{1}{2 c}\left(\frac{K y+M}{y-h(y)}\right)
$$

under the restriction $0<h(y)<y$.

Notice that (7) admits two positive solutions $h_{1}(y)$ and $h_{2}(y)$ such that the solutions satisfy $h_{1}(y) \leq h_{2}(y)$ for some $0<y<\infty$, where

$$
h_{1}(y)=\frac{1}{2}\left(1-\sqrt{1-\frac{2 K}{c}}\right) y-\frac{M}{c\left(1-\sqrt{1-\frac{2 K}{c}}\right)}
$$

is the minimal solution and

$$
h_{2}(y)=\frac{1}{2}\left(1+\sqrt{1-\frac{2 K}{c}}\right) y-\frac{M}{c\left(1+\sqrt{1-\frac{2 K}{c}}\right)}
$$

is the maximal solution with $c>2 K$ for $K>0$ and $M \geq 0$.

In the next section, using a comparison principle, a two-sided explicit estimate of the maximal solution $y \mapsto g_{*}(y)$ of (4) is given based on the maximal and minimal solutions of (7). Our main emphasis is on the explicit estimates obtained. This permits the generalization of the weighted maximal inequality (2) to those with explicit constants.

\section{Main results}

The next lemma will play an important role in the proof of our main result, which follows.

Lemma 5 (Comparison principle) Assume that condition (A) holds. Then the following positive bounds hold for the maximal solution $y \mapsto g_{*}(y)$ of $(4)$ :

$$
h_{1}(y) \leq g_{*}(y) \leq h_{2}(y)
$$

for some $0<y<\infty$, where $h_{1}(y)$ and $h_{2}(y)$ are given by (8) and (9), respectively.

Our main result is stated and proved in the next theorem.

Theorem 6 Let $B=\left(B_{t}\right)_{t \geq 0}$ be a standard Brownian motion starting at $x \geq 0$. Assume that condition (A) holds. Then the weighted maximal inequality

$$
\mathbf{E}_{x}\left[\left|B_{\tau}\right| H\left(\max _{0 \leq t \leq \tau}\left|B_{t}\right|\right)\right] \leq 2 K \mathbf{E}_{x}[\tau]+x H(x)+\frac{K}{2} x^{2}+M x+\frac{M^{2}}{2 K}
$$

holds for any stopping time $\tau$ for B satisfying $\mathbf{E}_{x}[\tau]<\infty$. 
Proof Using Lemma 2 and the two-sided estimate (10), it follows that

$$
\begin{aligned}
u(x, x)= & c x^{2}+x H(x)-2 c x g_{*}(x)+c\left(g_{*}(x)\right)^{2} \\
\leq & c x^{2}+x H(x)-c\left(1-\sqrt{1-\frac{2 K}{c}}\right) x^{2}+\frac{2 M x}{1-\sqrt{1-\frac{2 K}{c}}} \\
& +c\left(\frac{1}{2}\left(1+\sqrt{1-\frac{2 K}{c}}\right) x-\frac{M}{c\left(1+\sqrt{1-\frac{2 K}{c}}\right)}\right)^{2}
\end{aligned}
$$

with $c>2 K$ for $K>0$ and $M \geq 0$.

On the other hand, using the definition of the value function (3), we have

$$
\mathbf{E}_{x, x}\left[\left|B_{\tau}\right| H\left(\max _{0 \leq t \leq \tau}\left|B_{t}\right|\right)\right] \leq c \mathbf{E}_{x, x}[\tau]+u(x, x)
$$

for any stopping time $\tau$ for $B$ satisfying $\mathbf{E}_{x, x}[\tau]<\infty$.

Now using the upper bound in (12) in the right-hand side of the inequality (13) and passing to the limit as $c \downarrow 2 K$, we obtain the weighted maximal inequality (11). This completes the proof of the assertion.

Remark 7 Observe that our main result contains Theorem 1 as a special interesting case. This is in the case when $H(y)=y$, which is clearly a positive, continuous and differentiable function on $[0, \infty)$ with $H(0)=0$ and satisfying (6) with equality and $K=1$ and $M=0$.

\section{Illustrative example}

In this section, we shall now consider one example satisfying condition (A). Let $H(y)=\sin y$ for $0<y<\pi$. Clearly, $H$ is a positive, continuous and differentiable function for $0<y<\pi$. In this case, we have $\left|y H^{\prime}(y)\right| \leq y$ satisfying (6) with $K=1$ and $M=0$. Hence, the weighted maximal inequality (11) holds in the present example.

Note added in proof For a standard Brownian motion $B=\left(B_{t}\right)_{t \geq 0}$ starting at zero, in this case we have

$$
\mathbf{E}\left[\left|B_{\tau}\right| H\left(\max _{0 \leq t \leq \tau}\left|B_{t}\right|\right)\right] \leq 2 K \mathbf{E}[\tau]+\frac{M^{2}}{2 K}
$$

as a consequence of our main result. Now minimizing the right-hand side of the inequality (14) with respect to $K>0$ and assuming that $M>0$, it follows that

$$
\mathbf{E}\left[\left|B_{\tau}\right| H\left(\max _{0 \leq t \leq \tau}\left|B_{t}\right|\right)\right] \leq 2 M \sqrt{\mathbf{E}[\tau]}
$$

for any stopping time $\tau$ for $B$ satisfying $\mathbf{E}[\tau]<\infty$.

Competing interests

The author declares that he has no competing interests.

Received: 14 May 2015 Accepted: 12 October 2015 Published online: 02 November 2015 
References

1. Dubins, LE, Schwarz, G: A sharp inequality for sub-martingales and stopping times. Astérisque 157-158, 129-145 (1988)

2. Dubins, LE, Shepp, LA, Shiryaev, AN: Optimal stopping rules and maximal inequalities for Bessel processes. Theory Probab. Appl. 38, 226-261 (1993)

3. Peskir, G: Optimal stopping of the maximum process: the maximality principle. Ann. Probab 26, 1614-1640 (1998)

4. Lakshimikantham, V, Leela, S: Differential and Integral Inequalities. Vol. I. Academic Press, New York (1969)

5. McNabb, A: Comparison theorems for differential equations. J. Math. Anal. Appl. 119, 417-428 (1986)

6. Szarski, J: Differential Inequalities. Polish Scientific Publishers, Warszawa (1965)

7. Walter, W: Differential and Integral Inequalities. Springer, Berlin (1964)

Submit your manuscript to a SpringerOpen ${ }^{\circ}$ journal and benefit from:

- Convenient online submission

- Rigorous peer review

- Immediate publication on acceptance

- Open access: articles freely available online

- High visibility within the field

- Retaining the copyright to your article 